\title{
Restricted homotopies and focal conic textures
}

\author{
H.-R. Trebin and R. Kutka \\ Institut für Theoretische Physik der Universität, D-8400 Regensburg, Federal Republic of Germany
}

(Reçu le 9 juin 1981, accepté le 28 juillet 1981)

\begin{abstract}
Résumé. - C'est une étude de l'influence des conditions d'intégration sur la classification homotopique des singularités dans les phases lamellaires. Nous démontrons que des points singuliers d'indice quelconque existent pour des champs vectoriels, à trois dimensions qui ne possèdent pas de rotation. Des transitions continues entre des singularités du même indice sont interdites. Les défauts circulaires des systèmes focaux sont une manifestation d'une homotopie restreinte.
\end{abstract}

\begin{abstract}
The effects of integrability conditions on the homotopic defect classification of layered systems are investigated. It is shown, that for irrotational vector fields in three dimensions point defects of any index can be realized. Continuous transitions between defects of the same index are, however, impeded. The circular defect lines of focal conic textures are a manifestation of such a restricted homotopy.
\end{abstract}

With an ordered medium, like a ferromagnet, a superconductor, or a nematic liquid crystal, is associated an order parameter, which in the above examples is a unit vector, a phase or a director, respectively. Defects are sites in space where the order parameter is singular. The existence of stable defects is derived from topological properties of the order parameter space (a sphere, a uni circle in the complex plane, or the projective plane in the present examples). In the homotopic defect classification, as introduced into condensed matter physics by Toulouse and Kléman [1], and Volovik and Mineev [2], the set of topologically different line (point) defects in three dimensional space corresponds to the set of inequivalent closed contours (closed surfaces) in order parameter space. Two contours (surfaces) are equivalent - and belong to the same homotopy class - if they can be deformed continuously one into the other. The classification was also applied to systems of broken translational symmetry [2,3], but it was noted that eventually integrability conditions have to be taken into account [3]. As a consequence of this restraint it was assumed [4] that :

i) not every defect predicted by the topological classification can be realized as a single defect state ;

ii) there exist defect states corresponding to a particular homotopy class which cannot be deformed continuously one into the other under the restriction that at every stage of the deformation process the integrability conditions be satisfied.

The second point has been confirmed by Poénaru [5] with an example from the theory of foliations. Here we will first comment an example supporting the first point and then present two $n=1$ point defects of smectic-A liquid crystals which, under a simple integrability condition, are not any more topologically equivalent. We will show that the focal conic textures of smectic-A liquid crystals can be viewed as result of a restrained homotopy.

Whereas dislocations of layered systems are singularities in the phase of the matter wave, disclinations and point defects are singularities in the layer normals. We will describe the layers as surfaces $\varphi(\mathbf{r})=$ const of a smooth scalar function defined everywhere in physical space. Point defects are isolated singularities (zeroes) of the gradient field $\nabla \varphi$. In this model the investigation of point defects in layered systems reduces to the study of vector fields subjected to the integrability condition that they be irrotational.

Point defects of two-dimensional vector fields in the plane are labeled by the index $n$ which measures how many times the vector turns around as one follows the field along a closed loop surrounding the defect. It has been pointed out by Mermin [4] and rigorously proved by Poénaru [5], that in two dimensions an irrotational vector field with an isolated 
singularity (zero) of index larger than one does not exist. An analogous statement does not hold for a three-dimensional irrotational vector field $\mathbf{w}$ in threespace. In this case the index of a point singularity counts how often the normed vector

$$
\mathbf{w}^{0}(\mathbf{r})=\mathbf{w}(\mathbf{r}) /\|\mathbf{w}(\mathbf{r})\|
$$

sweeps over the unit sphere as one moves over a closed surface $S$ surrounding the point. The index coincides with the degree of the mapping [6 $\mathbf{w}^{0}$ on $S$. To construct a point defect of arbitrary index one starts from a scalar function $\varphi(x, y)$ in two dimensions whose gradient field has an isolated singularity of index $n$ at the origin. The function is extended to three dimensions by forming $\psi_{ \pm}(x, y, z)=\varphi(x, y) \pm \frac{1}{2} z^{2}$. An investigation of the degree of the mapping of $\nabla \psi_{ \pm} /\left\|\nabla \psi_{ \pm}\right\|$on a sphere about the origin reveals that the singularity of $\nabla \psi_{ \pm}$is of index $+n$ for $\psi_{+}$, and $-n$ for $\psi_{-}$. Since fields $\nabla \varphi$ for any negative integer exist, any homotopy class can be realized for irrotational vector fields in the three-dimensional case.

Examples for the preceding construction are the sink and the source, which are the gradient fields of $\varphi_{0}=-\frac{1}{2}\left(x^{2}+y^{2}\right)$ and $\varphi_{2}=\frac{1}{2}\left(x^{2}+y^{2}\right)$. Both have a singularity of index $n=1$ at the origin. Upon extension to three dimensions they become the hyperbolic point, $\nabla \psi_{0}$, and the hedgehog, $\nabla \psi_{2}$, also both of index $n=1$, where $\psi_{0}=\frac{1}{2}\left(z^{2}-x^{2}-y^{2}\right)$ and

$$
\psi_{2}=\frac{1}{2}\left(x^{2}+y^{2}+z^{2}\right) .
$$

Although now for any index a corresponding defect state exists, the integrability condition obstructs homotopies between defects of the same index, as we are going to demonstrate for the hyperbolic point and the hedgehog (also denoted elliptic point) in three dimensions. Stated differently, the problem is to replace the core of an elliptic point by the core of a hyperbolic point such that the resulting vector field is irrotational with only one isolated singularity. In terms of the scalar functions we ask if, given the scalar field $\psi_{0}$ inside a sphere of radius $R_{0}$, and $\psi_{2}$ outside a sphere of radius $R_{1}, 0<R_{0}<R_{1}$, it is possible to find a function $\psi_{1}$ in the shell

$$
R_{0}<|\mathbf{r}|<R_{1}
$$

joining smoothly to $\psi_{0}$ and $\psi_{2}$ such that the gradient of the resulting function $\psi$ has but one singularity (zero). Assume that such a function exists and consider it on a ball of radius $R_{2}>R_{1}$. Wrap this ball around a three-sphere $S^{3}$ such that the origin touches the south-pole and all the points at the surface meet at the north-pole (just as in two dimensions a disk is wrapped around a sphere). Denote by $\psi$ the field $\psi$ transferred from the three-ball to the three-sphere. Then $\Psi$, defined on a compact manifold, has two isolated singularities. As there is a maximum at the north-pole, there must be a minimum at the south-pole, contradicting the fact that there is already a hyperbolic (saddle) point. Hence elliptic and hyperbolic pointy are topologically distinct. This example shows that for irrotational vector fields there must be a second quantum number distinguishing the fields with singularities of index $n=1$. This additional label is apparently the Morse index, which is the number of negative eigenvalues of the Hessian $\partial^{2} \psi_{k} /\left.\partial x_{i} \partial x_{j}\right|_{r=0}$ of $\psi_{k}, k=0,2$, and which is +2 for $k=0,0$ for $k=2$.

An irrotational vector field interpolating between the elliptic and hyperbolic field exists, however, if a closed line defect is taken into account in the shell $R_{0}<|\mathbf{r}|<R_{1}$. It is constructed from a combination of two sources $(n=1)$ and one crosspoint $(n=-1)$ in two dimensions, which are extended to the third dimension by rotation about the $z$-axis (Fig. 1). The irrotational vector field stands perpendicular to the defect ring. Note that the defect ring would disappear, if close to the ring the vectors were turned parallel to it (" escape to the third dimension "); but the curl of the evolving vector field would not be zero.

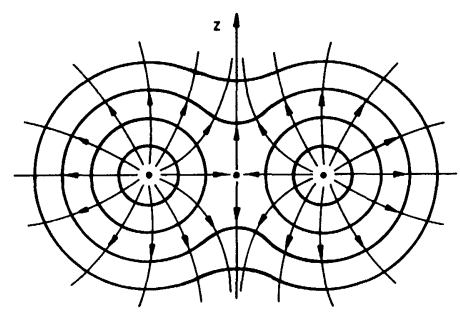

Fig. 1a. - Combination of two sources and one crosspoint in two dimensions, which by rotation about the $z$-axis becomes a three dimensional irrotational vector field with a point defect and a defect ring.

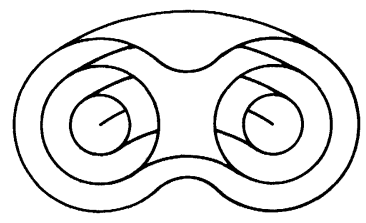

Fig. 1b. - Surfaces of constant value of the potential function which belongs to the irrotational vector field of figure $1 a$. Upon slight deformation near the $z$-axis the surfaces turn into Dupin cyclides.

By a slight deformation the layers perpendicular to the gradient field can be made equidistant, at the cost of an unstable line defect along the $z$-axis. The resulting structure is a special case of a focal conic texture [7], where the ellipse is degenerated to a circle, and the hyperbola to a straight line. Hence focal conic textures can be viewed as the layer system corresponding to an elliptic point whose core has been replaced by a hyperbolic point, and where the integrability conditions enforce the existence of a defect ring [8]. 
If this defect ring is shrunk to a point, the unstable line defect along the $z$-axis disappears, and a completely degenerate focal conic structure of concentric spheres arises, which is identical to the $n=1$ elliptic point defect of the homotopic defect classification for smectic-A liquid crystals. Indeed, if the integrability conditions are supplemented by the requirement of strict equidistance of the layers, this is the only realizable point defect.

The question persists, why focal conic domains with extended defect rings are most frequently observed, whereas the pure $n=1$ point defect is rarely seen. An answer can only be provided by studies of boundary conditions, growth, and energies of the textures. The elastic energy density for smectic-A liquid crystals of equidistant layers is

$$
f(\mathbf{r})=\frac{1}{2} K_{1}(\operatorname{div} \mathbf{n})^{2}=\frac{1}{2} K_{1}\left(\sigma_{1}+\sigma_{2}\right)^{2},
$$

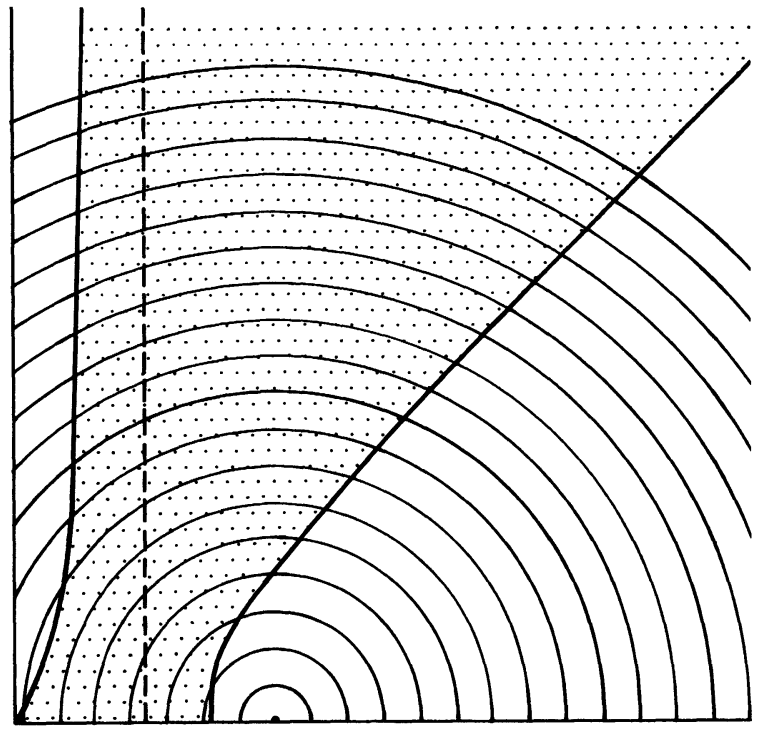

where $\mathbf{n}$ denotes the director, $\sigma_{1}$ and $\sigma_{2}$ the two main curvatures of the layers, and $K_{1}$ the splay elastic constant [9]. While around an elliptic point the energy density never vanishes, in a focal conic domain it is exactly zero on a cylinder of half the radius of the defect ring. In a large volume inside the ring, where the focal conic texture resembles the hyperbolic texture (Fig. 2), its energy density is lower than that of the elliptic point, since the main curvatures are of opposite sign. Outside the ring, it becomes elliptic, so that asymptotically the difference in energy density of both textures vanishes. On grounds of the local energy balance, which seems to us more important for the growth process than the total energy, a structure, that changes from the hyperbolic to the elliptic type, appears to be favoured over the pure elliptic point defect.

With the demonstration, that focal conic domains result from a combination of the fields of two topo-

Fig. 2. - Section of a focal conic texture. The elastic energy density in a focal conic domain is zero on the broken line and less than for the elliptic point defect in the shaded area.

logically inequivalent $n=1$ point defects, the incorporation of these textures into the homotopic defect classification scheme [10] has been elaborated in detail. The analysis shows that the integrability conditions lead to a refinement of the scheme which is expressed by additional topological quantum numbers.

Acknowledgements. - We have much profited from the mathematical advice of Prof. K. Jänich. One of us (H.-R. Trebin) has benefited from discussions with Prof. Louis Michel.

\section{References}

[1] Toulouse, G. and Kléman, M., J. Physique Lett. 37 (1976) L-149.

[2] VoloviK, G. E. and Mineev, V. P., Zh. Eksp. Teor. Fiz. 72 (1977) 2256 [Sov. Phys. JETP 45 (1977) 1186)].

[3] Kléman, M. and Michel, L., J. Physique Lett. 39 (1977) L-29 [Phys. Rev. Lett. 40 (1978) 1387].

[4] Mermin, N. D., Rev. Mod. Phys. 51 (1979) 591.

[5] Pó́naru, V., preprint (1981).

[6] See for instance Michel, L., Rev. Mod. Phys. 52 (1980) 617.
[7] Bouligand, Y., J. Physique 33 (1972) 525.

[8] This point of view is valid also for the general focal conic structure, which can be derived from the special case by continuous deformation.

[9] Kléman, M. and Parodi, O., J. Physique 36 (1975) 671

[10] It has been pointed out earlier by Prof. Kléman, M. (private communication) that in a large scale the focal conics are the point defects of smectic-A liquid crystals. 